ORIGINAL

\title{
Análisis de la morbimortalidad materna de las pacientes con preeclampsia grave, eclampsia y síndrome HELLP que ingresan en una Unidad de Cuidados Intensivos gineco-obstétrica
}

\section{E. Curiel-Balsera*, M.Á. Prieto-Palomino, J. Muñoz-Bono, M.J. Ruiz de Elvira, J.L. Galeas y G. Quesada García}

Unidad de Cuidados Intensivos Maternal, Hospital Regional Universitario Carlos Haya, Málaga, España

Recibido el 1 de marzo de 2011; aceptado el 9 de mayo de 2011

Disponible en Internet el 23 de junio de 2011

\author{
PALABRAS CLAVE \\ Preeclampsia grave; \\ Eclampsia; \\ Síndrome HELLP; \\ Unidad de cuidados \\ intensivos; \\ Complicaciones; \\ Mortalidad
}

\begin{abstract}
Resumen
Objetivo: Describir la incidencia y el perfil clínico y epidemiológico de las pacientes con preeclampsia grave que requieren ingreso en Cuidados Intensivos.

Diseño: Estudio observacional prospectivo de una serie de casos.

Ámbito: $\mathrm{UCl}$ específica de enfermedad gineco-obstétrica de 8 camas, perteneciente a un hospital universitario de nivel 3 , con una dotación de 55 camas de $\mathrm{UCl}$ en total.

Pacientes: Un total de 262 pacientes ingresadas por preeclampsia grave, eclampsia o síndrome HELLP.

Intervención: Análisis descriptivo de la población y de las complicaciones en $\mathrm{UCl}$ así como de la mortalidad intrahospitalaria.

Resultados: La edad media fue de 30,47 $\pm 5,7$ años, con una distribución diagnóstica al ingreso de $78 \%$ de pacientes con preeclampsia grave, $16 \%$ por síndrome HELLP y $6 \%$ por eclampsia, que sucedió en la semana gestacional $31,85 \pm 4,45$. El $63 \%$ de las pacientes fueron primigestas y presentaron escasa prevalencia de enfermedades previas. La tasa de complicaciones fue del $14 \%$ (fracaso cardiaco en $9 \%$, insuficiencia renal aguda en $5 \%$ y coagulopatía en $2 \%$ ).

La mortalidad materna fue 1,5\% (4 pacientes) y se relacionó con la no-primigestación, la presencia de complicaciones y un nivel superior de la transaminasa GOT a $71 \mathrm{mg} / \mathrm{dl}$.

Conclusiones: La preeclampsia grave tiene una baja tasa de mortalidad (1,5\%), no así de complicaciones $(14 \%)$ y se presenta con mayor frecuencia en nulíparas durante el tercer trimestre de gestación.

(C) 2011 Elsevier España, S.L. y SEMICYUC. Todos los derechos reservados.
\end{abstract}

\footnotetext{
* Autor para correspondencia.

Correo electrónico: emiliouci@telefonica.net (E. Curiel-Balsera).
} 


\section{KEYWORDS}

Severe preeclampsia;

Eclampsia;

HELLP syndrome; Intensive care unit; Complications; Mortality
Analysis of maternal morbidity and mortality among patients admitted to Obstetric Intensive Care with severe preeclampsia, eclampsia or HELLP syndrome

\begin{abstract}
Objective: To describe the incidence and clinical and epidemiological profile of patients with severe preeclampsia admitted to Intensive Care.

Design: A prospective, observational case series.

Setting: A specific obstetric 8-bed ICU belonging to a university hospital with a total of 55 ICU beds.

Patients: A total of 262 patients admitted due to severe preeclampsia, eclampsia or HELLP syndrome.

Intervention: Descriptive analysis of the population and complications in the ICU and hospital mortality.

Results: The mean patient age was $30.47 \pm 5.7$ years, with the following diagnóstico at admission: A total of $78 \%$ of the patients with severe preeclampsia, $16 \%$ with HELLP syndrome, and $6 \%$ with eclampsia, occurring in gestational week $31.85 \pm 4.45$. In turn, $63 \%$ of the patients were nulliparous and had a low prevalence of previous diseases. The global complications rate was $14 \%$ ( $9 \%$ heart failure, $5 \%$ acute renal failure and $2 \%$ coagulopathy).

Maternal mortality was $1.5 \%$ (4 patients), and was associated with non-nulliparous status, the presence of complications, and toast $>71 \mathrm{mg} / \mathrm{dl}$.

Conclusions: Severe preeclampsia has a low mortality rate $(1.5 \%)$, though the complications rate is considerable (14\%). The condition develops more often in nulliparous women during the third trimester of pregnancy.
\end{abstract}

(c) 2011 Elsevier España, S.L. and SEMICYUC. All rights reserved.

\section{Introducción}

La preeclampsia es un trastorno hipertensivo relativamente común durante el embarazo, de presentación progresiva, causa aún desconocida y que conlleva con frecuencia graves complicaciones maternales y perinatales. Se caracteriza por vasoespasmo y activación endotelial y se define por la presencia de hipertensión y proteinuria, después de la semana 20 de gestación.

La incidencia de esta dolencia es variable ya que en muchos estudios se han utilizado estimaciones basadas en muestras hospitalarias exclusivamente, lo que podría explicar las cifras utilizadas con relativa frecuencia de hasta el 5-10\%, dependiendo de los niveles asistenciales de los hospitales donde se haga el estudio. Se estima que alrededor del $7 \%$ de los embarazos desarrollarán una preeclampsia ${ }^{1}$, aunque esta puede ser mayor en entornos socioeconómicos más desfavorecidos y también en países donde hay más prevalencia de enfermedades cardiovasculares ${ }^{2}$. Aunque existen pocas estimaciones realizadas en España, las disponibles se sitúan entre el 1 y el $2 \%^{3}$.

El $5 \%$ de las preeclampsias evolucionan finalmente a eclampsia ${ }^{4}$, y hasta en un $19 \%$ pueden hacerlo como un síndrome HELLP, lo que se asocia con una mayor morbimortalidad $^{5}$.

Los resultados maternales adversos en la preeclampsia se deben, fundamentalmente, a una disfunción en el sistema nervioso central, hepático o renal (accidente cerebrovascular hemorrágico, rotura hepática o fracaso renal agudo), y al sangrado asociado a trombocitopenia. La preeclampsia-eclampsia es una de las 3 causas más frecuentes de mortalidad en la embarazada, junto a la enfermedad tromboembólica y a la hemorragia postparto ${ }^{6}$.
Los criterios de preeclampsia grave fueron establecidos por la Sociedad Española de Ginecología y Obstetricia en 2006, y marcan a un subgrupo de pacientes con mayor mortalidad maternofetal y un manejo más frecuente en unidades de cuidados intensivos ${ }^{7}$.

Nuestro objetivo ha sido describir la epidemiología y los factores de riesgo y evolutivos de las pacientes con preeclampsia grave, eclampsia y síndrome HELLP.

\section{Material y métodos}

Se ha realizado un estudio observacional, prospectivo, con todas las pacientes ingresadas en la Unidad de Cuidados Intensivos (UCI) Maternal del Hospital Materno Infantil, perteneciente al Complejo Hospitalario Carlos Haya de Málaga, por preeclampsia grave, eclampsia o síndrome HELLP, desde enero de 1999 hasta septiembre de 2008.

El Hospital Materno Infantil atiende a la mitad de la población de la ciudad de Málaga y es el único hospital público de la provincia dotado de Unidad de Cuidados Intensivos neonatales, por lo que es hospital de referencia para las pacientes con preeclampsia severa, eclampsia o síndrome HELLP cuando la edad gestacional está poco avanzada. La Unidad de Cuidados Intensivos se trata de una $\mathrm{UCl}$ específica de dolencia gineco-obstétrica de 8 camas, englobada en el H.R.U Carlos Haya, centro hospitalario de nivel III, con una dotación de 55 camas de UCI en total.

La preeclampsia grave se define por la presencia de cifras de presión arterial sistólica $\geq 140 \mathrm{~mm} \mathrm{Hg}$ o presión arterial diastólica $\geq 90 \mathrm{~mm} \mathrm{Hg}$ en dos o más tomas separadas al menos por 6 horas, junto con proteinuria $(\geq 300 \mathrm{mg}$ en orina de $24 \mathrm{~h}$ ) y alguno de los criterios de gravedad que se 
Tabla 1 Signos y síntomas de preeclampsia grave (al menos uno de los siguientes)

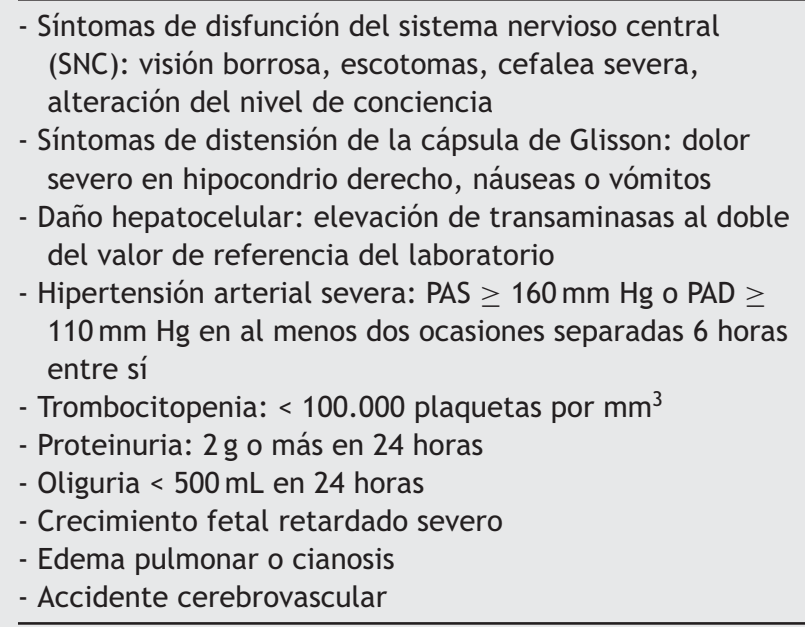

muestran en la tabla 1. Cuando sobre una preeclampsia se presentan convulsiones o coma sin otra causa que lo justifique, hablamos de eclampsia. Síndrome HELLP es el acrónimo de hemolisis-elevated liver enzimes and low platelets, que es otra forma de presentación de la preeclampsia grave.

Se registraron variables clínico-epidemiológicas, así como variables analíticas al ingreso o de manejo obstétrico. Se analizó la mortalidad materna y los factores relacionados con ella, durante el ingreso hospitalario por causa relacionada con la gestosis, así como los factores relacionados con las complicaciones, referidas al desarrollo de fracaso cardiaco (signos y síntomas de insuficiencia cardiaca aguda que obligaron al uso de oxigenoterapia o de ventilación mecánica invasiva o no), coagulopatía (disminución de la actividad de protrombina por debajo del 50\% o del TPTA al doble del control, o presencia de sangrado externo o interno que inestabilizó a la paciente y requirió el uso de hemoderivados o cirugía para su control) o fracaso renal agudo (por elevación al doble de las cifras de creatinina basal en el momento del ingreso o de oligoanuria que obligó a iniciar técnicas de reemplazo extrarrenal). Asimismo, se analizó el promedio de estancia en $\mathrm{UCl}$ y los factores determinantes de la misma.

La finalización de la gestación se indicó por la presencia de complicaciones graves maternales, por existencia de sufrimiento fetal, o ante la ausencia de crecimiento durante, al menos, una semana pese al tratamiento adecuado. La decisión de la indicación y la elección del momento se realizó conjuntamente con el Servicio de Ginecología.

Se informó a las pacientes y/o familiares de la naturaleza de la investigación y del uso que se iba a hacer de la misma. Se garantizó en todo momento la confidencialidad, así como que toda la información solo sería utilizada para los fines especificados.

\section{Análisis estadístico}

Las variables cuantitativas se expresaron como media \pm desviación estándar; las variables cualitativas como frecuencia (porcentaje). La comparación entre grupos se realizó, en el caso de las variables cuantitativas, con el test de la T de Student, o en su caso, con el test no paramétrico de Mann-Whitney, y el test ANOVA para la comparación de más de 2 grupos. En el caso de variables cualitativas, se utilizó el test de la chi cuadrado $\left(\mathrm{Chi}^{2}\right)$ o la prueba exacta de Fisher.

Se realizó análisis multivariante mediante regresión logística, para identificar predictores de mortalidad y desarrollo de complicaciones, y regresión lineal múltiple para los factores predictores de estancia en $\mathrm{UCl}$. Se incluyeron en el análisis las variables significativas en el estudio bivariante.

En todos los casos se consideró la existencia de significación estadística a aquella con $\mathrm{p}<0,05$ y el intervalo de confianza se estableció para el $95 \%$ de los casos.

\section{Resultados}

Se incluyeron 262 mujeres con preeclampsia grave, con un promedio de 30,47 $\pm 5,7$ años de edad, entre los 65.503 partos realizados en nuestro centro. La incidencia de preeclampsia grave en nuestro estudio fue 4/1.000 partos. Esto representó que el $0,37 \%$ de las pacientes ingresadas en el hospital tras dar a luz, requirieron ingreso en $\mathrm{UCl}$ por preeclampsia grave, eclampsia o HELLP.

La distribución diagnóstica al ingreso fue: 204 (78\%) con preeclampsia grave, 42 (16\%) con síndrome HELLP y 16 (6\%) con eclampsia, que sucedió en la semana gestacional 31,8 $\pm 4,4$. El $63 \%$ de las pacientes fueron primigestas y presentaron escasa prevalencia de tabaquismo $(3,44 \%)$, obesidad $(10,3 \%)$ o hipertensión arterial previa $(17,8 \%)$. La finalización de la gestación sucedió por cesárea en el $90 \%$ de los casos.

En la tabla 2 se muestran algunas variables clínicas y epidemiológicas según los motivos de diagnóstico.

La edad gestacional de las mujeres con eclampsia $(35,6 \pm 5)$ fue significativamente superior a la de las gestantes con preeclampsia $(32 \pm 4,2)$ y con síndrome HELLP $(30,9 \pm 3,8),(p=0,004$ y $p=0,001$, respectivamente).

Un 14\% presentó alguna complicación, siendo por orden de frecuencia, el fracaso cardiaco en 23 pacientes (9\%), insuficiencia renal aguda en 14 pacientes (5\%) y coagulopatía en 6 casos (2\%). La tabla 3 expresa parámetros clínicos y analíticos de las pacientes según el desarrollo de complicaciones o no. No se encontraron diferencias significativas en el desarrollo de complicaciones según el diagnóstico al ingreso como se aprecia en la figura 1.

El promedio de presión arterial sistólica (PAS) fue $171,7 \pm 82,7$ mientras que el de diastólica (PAD) fue $99 \pm 15$. No encontramos asociación entre el promedio de cifras de PAS-PAD y el desarrollo de complicaciones.

La mortalidad materna fue $1,5 \%$ (2 pacientes diagnosticadas de preeclampsia grave y 2 de ellas con síndrome HELLP). La primigestación fue un factor protector de mortalidad materna $(p=0,008)$, mientras que el promedio de cifras de transaminasas se relacionó con mayor mortalidad $(p=0,0001)$.

La estancia media en la UCI fue 5,6 $\pm 3,4$ días. Las pacientes que sufrieron alguna complicación presentaron mayor estancia media en la unidad $5,3 \pm 2,8$ vs $8 \pm 5(p=0,007)$, 
Tabla 2 Características clínico-demográficas según diagnóstico al ingreso

\begin{tabular}{llll}
\hline & Preeclampsia $(\mathrm{n}=203)$ & Eclampsia $(\mathrm{n}=16)$ & Síndrome HELLP $(\mathrm{n}=43)$ \\
\hline Edad & $31 \pm 5$ & $29 \pm 7$ & $30 \pm 6$ \\
Primigestación & $124(61,1 \%)$ & $11(68,8 \%)$ & $30(69,8 \%)$ \\
Obesidad & $23(11,3 \%)$ & $3(18,8 \%)$ & $1(2,3 \%)$ \\
Hipertensión arterial & $40(19,7 \%)$ & $0(0 \%)$ & $6(14 \%)$ \\
Tabaquismo & $7(3,4 \%)$ & $1(6,3 \%)$ & $1(2,3 \%)$ \\
Semana gestacional al ingreso & $32 \pm 4$ & $36 \pm 5$ & $31 \pm 4$ \\
Estancia en UCl (días) & $6 \pm 4$ & $7 \pm 4$ & $6 \pm 3$ \\
\hline
\end{tabular}

Tabla 3 Datos clínicos y analíticos según desarrollo de complicaciones

\begin{tabular}{llll}
\hline & No complicaciones $(\mathrm{n}=225)$ & Complicaciones $(\mathrm{n}=37)$ & Valor $\mathrm{p}$ \\
\hline Edad & $31 \pm 6$ & $30 \pm 6$ & 0,74 \\
Obesidad & $26(11,3 \%)$ & $1(2,7 \%)$ & 0,101 \\
Tabaquismo & $7(3,1 \%)$ & $2(5,4 \%)$ & 0,47 \\
Primigestación & $142(63,1 \%)$ & $23(62,4 \%)$ & 0,91 \\
Semana gestacional al ingreso & $32 \pm 5$ & $32 \pm 4$ & 0,587 \\
PAS & $167 \pm 24$ & $168 \pm 19$ & 0,746 \\
PAD & $99 \pm 16$ & $99 \pm 10$ & 0,812 \\
Hemoglobina & $11 \pm 2$ & $10 \pm 2$ & 0,005 \\
Plaquetas & $179.644 \pm 94.549$ & $160.811 \pm 70.532$ & 0,24 \\
GOT (AST) & $78 \pm 114$ & $143 \pm 417$ & 0,38 \\
GPT (ALT) & $81 \pm 98$ & $104 \pm 228$ & 0,57 \\
Acido úrico & $6,2 \pm 1,3$ & $6,3 \pm 1,9$ & 0,66 \\
Estancia (días) & $5 \pm 3$ & $8 \pm 5$ & 0,007 \\
\hline
\end{tabular}

GOT: aspartato amino transferasa; GPT: alanino amino transferasa; PAD: presión arterial diastólica; PAS: presión arterial sistólica.

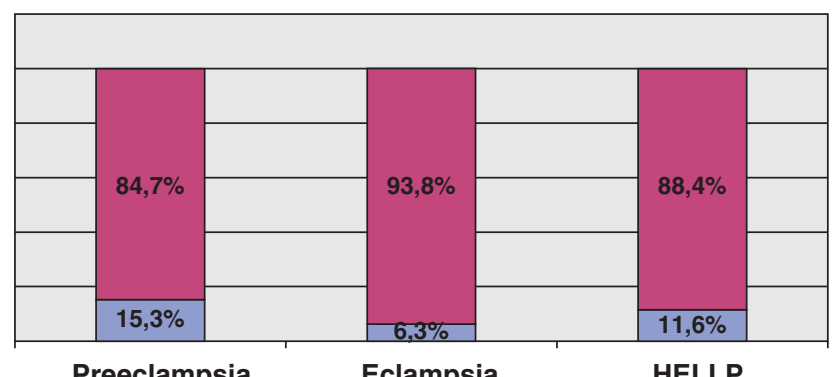

Preeclampsia
HELLP

\section{$\square$ Complicaciones $\square$ No complicaciones}

Figura 1 Complicaciones en función del diagnóstico de ingreso.

lo cual se mantuvo de manera aislada con el fracaso cardiaco y la insuficiencia renal aguda $(p=0,004$ y $p=0,0001$, respectivamente), pero no así con la coagulopatía $(p=0,7)$.

El análisis multivariante de la mortalidad materna en las tres entidades mostró a la primigestación, la presencia de complicaciones y un nivel superior de la transaminasa GOT a $71 \mathrm{mg} / \mathrm{dl}$ como factores explicativos de la mortalidad materna (tabla 4).

Los predictores independientes de estancia en $\mathrm{UCI}$ fueron las cifras de hemoglobina ( $p=0,005$, IC 95\% $-0,6,-0,1)$ y la edad gestacional en el momento del parto $(p=0,001$, IC 95\% $-0,2,-0,07)$. La estancia en UCI fue el único predictor independiente del desarrollo de complicaciones, $(p=0,0001, \mathrm{OR}$ 0,8 , IC 95\% 0,7-0,9).

Tabla 4 Análisis multivariante de la mortalidad materna

\begin{tabular}{lrrrrr}
\hline & $\beta$ & Sig. & OR & \multicolumn{1}{c}{ IC 95,0\% } & Superior \\
\hline Primigestación & & & & Inferior & 0,526 \\
GOT(AST) $\leq 71 \mathrm{mg} / \mathrm{dl}$ & $-5,283$ & 0,026 & 0,005 & 0,000 & 0,834 \\
Presencia de complicaciones & $-4,402$ & 0,041 & 0,012 & 0,000 & 45,835 \\
Constante & 3,130 & 0,041 & 22,872 & 1,141 & \\
\hline
\end{tabular}

$\beta$ : coeficiente beta; GOT (AST): aspartato aminotransferasa; PAS: presión arterial sistólica. 


\section{Discusión}

La preeclampsia grave, eclampsia y síndrome HELLP presentan una baja incidencia (4 casos/1.000 partos) y una mortalidad baja $(1,5 \%)$, si bien la tasa de complicaciones no es desdeñable $(14 \%)$, en un entorno de manejo de cuidados intensivos.

La mortalidad maternal por causas obstétricas varía según el entorno. Así, en países occidentales oscila entre el $0,2 \%$ de la serie de Zeeman et al, constituida exclusivamente con pacientes gestósicas ${ }^{8}$, y el $3 \%$ de la de Gilbert ${ }^{9}$. En países menos desarrollados, la mortalidad materna alcanza cifras muy elevadas: $33 \%$ en la serie de Okafor de Nigeria ${ }^{10}, 22 \%$ en un registro de India ${ }^{11}$, casi el $20 \%$ de una serie tunecina ${ }^{12}$ o un $10 \%$ de la serie turca de Demirkiran ${ }^{13}$, todas ellas publicadas en la década actual. En nuestro medio, el grupo de Olarra ${ }^{14}$ constató, en un estudio retrospectivo, una mortalidad materna del $7,5 \%$ en una serie de 149 gestantes graves ingresadas a lo largo de 7 años, de las que 50 ingresaron por preeclampsia.

Nuestra serie, referida exclusivamente a pacientes con preeclampsia grave, registró una mortalidad materna del $1,5 \%$, porcentaje similar a los obtenidos por Zeeman ${ }^{8}$ y otros autores en países occidentales. Dado el bajo número de pacientes fallecidas, no se pueden extraer conclusiones válidas.

El síndrome HELLP comporta un riesgo aumentado de mortalidad materno-fetal. Un hospital de nuestro entorno registró una mortalidad maternal del $1,8 \%{ }^{15}$, mientras que en la serie más numerosa de Sibai fue del $1 \%{ }^{16}$.

Los criterios de ingreso obstétricos en unidades especializadas de cuidados críticos varían según la prevalencia de determinadas enfermedades, áreas geográficas, definiciones operativas de morbilidad, criterios de ingreso, y protocolos de manejo y tratamiento, entre otros aspectos relevantes, aunque se estima una utilización de cuidados intensivos obstétricos entre el 1-3\% de los partos, especialmente por preeclampsia grave y hemorragias masivas ${ }^{8}$. En nuestro hospital, un $0,37 \%$ de las mujeres atendidas en el Hospital durante el periodo de estudio requirieron ingreso en la $\mathrm{UCl}$ por preeclampsia grave, lo que representa un porcentaje similar al de países de nuestro entorno ${ }^{17}$.

Las complicaciones durante su estancia en $\mathrm{UCl}$ se relacionan con peor pronóstico, de manera que en el análisis multivariante fue la variable con mayor peso específico. La incidencia de fallo renal agudo en la preeclampsia no está claramente definida. En una reciente revisión ${ }^{18}$ de la insuficiencia renal de origen obstétrico, se apunta a la preeclampsia como responsable del $20,9 \%$ de los fallos renales, mientras que en otra serie analizada, que incluyó 31 casos de gestaciones complicadas con fallo renal, el $58 \%$ fue debido a una preeclampsia, que originó una necrosis tubular aguda, y la mitad de los casos requirió hemodiálisis durante su hospitalización.

Otro estudio analizado en la anterior revisión constató que un $5 \%$ de las gestosis se complicaron con fracaso renal agudo, llegando al 7,3\% en el subgrupo de pacientes con HELLP. Por otra parte, la mayoría de los casos registrados en la literatura recuperaron una función renal normal en un periodo de tiempo variable, como así sucedió en nuestras pacientes $^{18}$.
Entre los cambios del sistema cardiovascular que se producen en la preeclampsia destacan la reducción del gasto cardiaco y el aumento de la resistencia vascular periférica. El estudio de Terrone ${ }^{19}$ constató la presencia de morbilidad cardiaca en un 7,6\% al analizar una serie numerosa de mujeres con preeclampsia y síndrome HELLP. En sus conclusiones se reflejó que en ese subgrupo de gestantes predominó la cesárea como modo de finalización del parto, la presencia de neonatos de bajo peso, una mayor hipertensión arterial y la existencia de anomalías analíticas. El fallo cardiaco y el edema pulmonar se registraron con similar frecuencia en la preeclampsia y en el síndrome HELLP. Nuestra serie registró un porcentaje ligeramente superior (9\%, 23 casos) de gestosis complicadas con insuficiencia cardiaca. La presencia de fracaso cardiaco sí conllevó una mayor estancia, y significativa, en la $\mathrm{UCI}$ con relación a las pacientes que no lo presentaron.

El análisis multivariante de las complicaciones maternas tan solo identifica la estancia prolongada en UCl como predictor, si bien esta pudo ser la consecuencia de la presencia de complicaciones. Por tanto, en este caso, el modelo multivariante no da una respuesta satisfactoria a la cuestión planteada.

La estancia media de nuestras pacientes fue de 5 días, similar a lo descrito por otros autores ${ }^{8}$, sin diferencias entre las tres entidades diagnósticas. Sí que parece razonable pensar que estancias medias más prolongadas son sinónimo de peor evolución y mayor tasa de complicaciones, como así se pudo comprobar. El análisis estadístico identificó como variables independientes de la estancia en la $\mathrm{UCl}$ el nivel de hemoglobina y la edad gestacional durante el parto, de manera que una menor concentración de hemoglobina o una semana gestacional baja ocasionaron una estancia en la Unidad más prolongada, en relación con las mayores necesidades de monitorización y tratamiento. En las series que analiza Zeeman la estancia más prolongada se debió a la necesidad temporal de ventilación mecánica, de transfusión de hemoderivados o de soporte inotrópico.

La asociación gestosis-primiparidad sigue siendo muy controvertida ${ }^{20,21}$. En nuestra serie, la primigestación fue un factor prevalente $(62,9 \%)$, tal y como se ha descrito en otros trabajos ${ }^{21}$, pero es posible que la primigestación esté sobreestimada como factor de riesgo de preeclampsia ya que, ante el temor de padecer esta dolencia en sucesivas ocasiones, las pacientes autolimiten nuevas gestaciones.

Un reciente metaanálisis ${ }^{22}$, que revisó 26 estudios publicados entre 1966 y 2005, concluyó que el riesgo de preeclampsia era 2,42 veces mayor en una primípara que en una multípara, planteándose diferencias en la respuesta inmunológica de las primíparas respecto a las multíparas, del perfil angiogénico o del patrón de reactividad de resistencia insulínica como factores etiopatogénicos.

El programa de investigación Pre-eclampsia Integrated Estimate of RiSk (PIERS), que surgió con el propósito de desarrollar una escala que sirviese de guía de práctica clínica y de toma de decisiones en casos de riesgo maternal asociado al diagnóstico de preeclampsia, estableció que la mayoría de los criterios de severidad de la enfermedad predecían escasamente la morbimortalidad maternofetal. En cambio, la valoración y vigilancia estandarizada de las pacientes con preeclampsia grave sí reducían el riesgo maternal ${ }^{23}$. Es por ello por lo que la vigilancia y monitorización de 
estas pacientes en Cuidados Intensivos pueda detectar de forma precoz la aparición de complicaciones con un beneficio secundario sobre la mortalidad.

Las limitaciones de nuestro trabajo se basan en las pocas conclusiones obtenidas sobre la mortalidad por la baja tasa de la misma $(1,5 \%)$ y en la falta de especificidad de algunas de las definiciones, como la de fracaso renal, que en los últimos años ha cambiado, sin poder adaptarnos a clasificaciones más actuales como la escala RIFLE o la AKIN.

Entre las ventajas se encuentran el tamaño muestral, tratándose de la serie más grande sobre gestosis grave publicada en nuestro país, y el manejo especializado en una UCI de un hospital de tercer nivel dedicado de forma exclusiva a enfermedad gineco-obstétrica.

\section{Conflicto de intereses}

Los autores declaran no tener ningún conflicto de intereses.

\section{Bibliografía}

1. Bhattacharya S, Campbell DM. The incidence of severe complications of preeclampsia. Hypertens Pregnancy. 2005;24:181-90.

2. Kaaja R, Kinnunen T, Luoto R. Regional differences in the prevalence of pre-eclampsia in relation to the risk factors for coronary artery disease in women in Finland. Eur Heart J. 2005;26:44-50.

3. Gratacós E, Cabero Ll. Diagnóstico de preeclampsia y eclampsia. Jano. 2000;58:61-3.

4. Pregnancy-induced hypertension. Professional Guide to Diseases. $8 .^{a}$ ed. China: Springhouse ed. Lippincott Williams \& Wilkins; 2005.

5. Abroug F, Boujdaria R, Nouira S, Abroug S, Souissi M, Najjar MF, et al. Hellp syndrome: incidence and maternal-fetal outcome. A prospective study. Intensive Care Med. 1992;18:274-7.

6. CDC. Health, United States, 2008. Hyattsville, MD: National Center for Health Statistics; 2008.

7. Rubio Lorente AM, González López AB, González Mirasol E, González de Merlo G. Morbimortalidad materna y fetal en pacientes con preeclampsia grave. Prog Obstet Ginecol. 2011;54:4-8.

8. Zeeman GG. Obstetric critical care: A blueprint for improved outcomes. Crit Care Med. 2006;34 suppl.:208-14.

9. Gilbert TT, Smulian JC, Martin AA, Ananth CV, Scorza W, Scardella AT. Obstetric admissions to the intensive care unit: Outcomes and severity of illness. Obstet Gynecol. 2003;102:897-903.

10. Okafor UV, Aniebue U. Admisión pattern and outcome in critical care obstetric patients. Int J Obstet Anesth. 2004;13:164-6.

11. Karnad DR, Lapsia V, Krishnan A, Salvi VS. Prognostic factors in obstetric patients admitted to an Indian intensive care unit. Crit Care Med. 2004;32:1294-9.

12. Chelli D, Dimassi K, Zouaoui B, Sfar E, Chelli H, Chennoufi MB. Evolution of maternal mortality in a level 3 Tunisian maternity from 1998 to 2007. J Gynecol Obstet Biol Reprod (Paris). 2009;38:655-61.

13. Demirkirian O, Dikmen Y, Utku T, Urkmez S. Critically ill obstetric patients in the intensive care unit. Int J Obstet Anesth. 2003;12:266-70.

14. Olarra J, Longarela AM, Suárez L, Palacio FJ. Critically ill obstetric patients treated in an ICU. Chest. 2002;121:2077.

15. Vallejo I, Miranda ML, Stiefel P, Pamies E, Marenco ML, Castro D, et al. Características clinicobiológicas de un grupo de 54 gestantes con síndrome HELLP. Med Clin (Barc). 2004;122:259-61.

16. Sibai BM, Ramadan MK, Usta I, Salama M, Mercer BM, Friedman SA. Maternal morbidity and mortality in 442 pregnancies with hemolysis, elevated liver enzymes, and low platelets (HELLP syndrome). Am J Obstet Gynecol. 1993;169:1000-6.

17. Zhang WH, Alexander S, Bouvier-Colle MH, Macfarlane A. Incidence of severe pre-eclampsia, postpartum haemorrhage and sepsis as a surrogate marker for severe maternal morbidity in a European population-based study: the MOMS-B survey. BJOG. 2005;112:89-96.

18. Mirza FG, Cleary KL. Pre-eclampsia and the kidney. Semin Perinatol. 2009;33:173-8.

19. Terrone DA, Isler CM, May WL, Magann EF, Norman PF, Martin JN. Cardiopulmonary morbidity as a complication of severe preeclampsia HELLP syndrome. J Perinatol. 2000;20:78-81.

20. Robillard PY, Dekker G, Chaouat G, Hulsey TC. Etiology of preeclampsia: maternal vascular predisposition and couple disease-mutual exclusion or complementarity? J Reprod Immunol. 2007;76:1-7.

21. Hernández-Díaz S, Toh S, Cnattingius S. Risk of pre-eclampsia in first and subsequent pregnancies: prospective cohort study. BMJ. 2009;338:b2255.

22. Luo ZC, An N, Xu HR, Larante A, Audibert F, Fraser WD. The effects and mechanisms of primiparity on the risk of preeclampsia: a systematic review. Paediatr Perinat Epidemiol. 2007;21 Suppl 1:36-45.

23. Von Dadelszen P, Menzies J, Payne B, Magee L. Predicting adverse outcomes in women with severe pre-eclampsia. Semin Perinatol. 2009;33:152-7. 